\title{
Implicaciones clínicas del diagnóstico diferencial temprano entre Retraso de Lenguaje (RL) y Trastorno Específico del Lenguaje (TEL)*
}

\author{
Clinical Implications of early differential diagnostic between \\ Language Delay (LD) and Specific Language Impairment (SLI)
}

Recibido: abril 23 de 2010 | Revisado: noviembre 10 de 2010 | Aceptado: febrero 14 de 2011

\author{
VíCTOR ACOSTA RODRÍGUEZ** \\ ANA MORENO SANTANA *** \\ ÁNGELES AXPE CABALLERO ***** \\ Facultad de Psicología, Universidad de \\ La Laguna, Islas Canarias, España
}

SICI: 2011-2777(201203)11:1<279:IRLTEL>2.0.CO;2-W

Para citar este artículo: Acosta, V., Moreno, A. \& Axpe, A. (2012). Implicaciones clínicas del diagnóstico diferencial temprano entre Retraso de Lenguaje (RL) y Trastorno Específico del Lenguaje (TEL). Universitas Psychologica, 11(1), 279-291.

* Agradecemos la ayuda recibida a través del Proyecto de Investigación La intervención integrada en el lenguaje y la lectura emergente con niños con Trastorno Específico de Lenguaje (TEL), financiado por el Ministerio de Ciencia e Innovación, con referencia SEJ2007-67037/EDUC.

** Catedrático de Educación Especial y Logopedia, Presidente de la Asociación Española de Logopedia, Foniatría y Audiología. Facultad de Psicología, Departamento de Didáctica e Investigación Educativa, Universidad de La Laguna, Islas Canarias. C/. Delgado Barreto s/n 38204 , La Laguna (S/C de Tenerife). E-mail: vacosta@ ull.es

*** Profesora Titular de Didáctica y Organización Escolar. Departamento de Didáctica e Investigación Educativa, Islas Canarias. C/. Delgado Barreto s/n, 38204 La Laguna (S/C de Tenerife).

***** Profesora Contratada Doctora de Métodos de Investigación y Diagnóstico en Educación. Departamento de Didáctica e Investigación Educativa, Islas Canarias. C/. Delgado Barreto s/n, 38204 La Laguna (S/C de Tenerife).

\section{RESUMEN}

El objetivo de este trabajo es analizar las diferencias que, desde un punto de vista clínico, tienen los conceptos de Retraso de Lenguaje (RL) y Trastorno Específico del Lenguaje (TEL). Para tal fin, se seleccionó una muestra de seis sujetos con RL y otros seis con TEL, a través del uso de instrumentos tanto estandarizados como en forma de tareas. Mientras que los niños con RL no recibieron tratamiento alguno, los TEL fueron sometidos a un programa de intervención que perseguía, por un lado, favorecer el desarrollo de la comprensión y la producción lingüística y, por otro, estimular el progreso de habilidades básicas para la iniciación a la lectura, especialmente el desarrollo narrativo y el procesamiento fonológico. Los contenidos del programa se han secuenciado en orden creciente de complejidad cognitiva. Con el fin de comprobar las semejanzas o diferencias de los sujetos en su desarrollo lingüístico, atendiendo a su grupo de pertenencia, se utilizó la $U$ de MannWhitney. Los resultados obtenidos muestran diferencias estadísticamente significativas entre ambos grupos. Así, se destaca una evolución siempre favorable de los sujetos con RL, sin que sean sometidos a intervención alguna, todo lo contrario de lo que ocurre con los niños con TEL. En consecuencia, los datos apuntan a que no tiene por qué haber un recorrido desde el RL hasta el TEL, tratándose de categorías diagnósticas diferenciadas.

Palabras clave autores:

Trastorno del lenguaje, retraso del lenguaje, evaluación e intervención en el lenguaje. Palabras clave descriptores:

Logopedia, detección y diagnóstico, intervención temprana.

\section{A B S T R A C T}

The aim of this paper is to analyze the differences between Language Delay (LD) and Specific Language Impairment (SLI) concepts from a clinical point of view. The selected sample consisted of 6 LD and 6 SLI individuals, who were chosen with standardized tools and qualitative tasks. LD children had not any treatment; meanwhile SLI children underwent an intervention program which pursued two aims: on one hand, to improve the development of linguistic comprehension and production and on the other hand, to encourage the progress of basic skills for literacy, focusing on narrative development and phonological processing. The contents of the program have been sequenced in increasing order of cognitive complexity. The $\mathrm{U}$ of Mann-Whitney was used to check the similarities and differences of the children' linguistic development according to their diagnosis. Results 
show important differences between both groups. A positive progress of LD children stand outs, although they didn't undergo an intervention program. Meanwhile, just the opposite happens with SLI children. These data make us think that there is not an obligatory sequence from LD to SLI children. Therefore, we regard them as different categories.

Key words authors:

Language impairment, language delay, assessment and

intervention.

Key words plus:

Speech and language therapist, screening and diagnostic, early intervention.

\section{Introducción}

Hace ya algunos años que el Trastorno Específico del Lenguaje (TEL) viene ocupando un lugar destacado en la investigación, tanto básica como aplicada, relacionada con los problemas concretos que ocasiona tanto en el lenguaje como en las áreas relacionadas con este. Uno de los objetivos principales ha sido tratar de describir de la manera más precisa este trastorno. En síntesis, y siguiendo los criterios clínicos de diversos autores (Bishop, 1997; Hincapié et al., 2008; Leonard, 1998; Pérez \& Serra, 1998; Serra, 1997; Serra \& Conti-Ramsden, 1998), solo se considera un TEL cuando no se detecta la presencia de otros problemas asociados, sean de tipo emocional, cognitivo, motriz o sensorial, que puedan haberlo causado. Paralelamente, y a medida que se ha incrementado el conocimiento acerca de este trastorno, se ha ido generando también un volumen importante de discusión. Sin lugar a dudas, uno de los debates más interesantes y, al mismo tiempo, controvertidos ha sido el de las posible diferenciación o no, entre el binomio Retraso de Lenguaje (RL) y TEL. En esta última línea de reflexión, ocupa un lugar destacado la identificación temprana de niños con problemas de lenguaje a los que se ha ido definiendo de manera distinta. Así, entre 18 y 20 meses de edad, se les ha puesto la etiqueta de hablantes tardíos o retraso inicial de lenguaje (late talkers), para distinguir un grupo de niños cuya característica más sobresaliente es un empobrecimiento lingüístico, puesto de manifiesto a través de un retraso considerable a la hora de iniciar el habla -que puede resultar anómala, en determinadas ocasiones-, junto con un volumen de vocabulario muy limitado y una ausencia de es- tructuras con dos palabras. El RL (language delay) se distingue por llegar, entre los 24-36 meses, con un vocabulario también muy reducido (no superando las cien primeras palabras), así como con un desarrollo muy básico de la sintaxis y de la morfología (a la hora de combinar dos o más palabras, y tardanza en aparecer la flexión morfológica productiva). En general, se está hablando de sujetos que exhiben un lenguaje expresivo muy simplificado y una cierta afectación en su habla. Estas peculiaridades situarían a los dos tipos anteriores bajo la etiqueta de grupos de riesgo y, lo que es más importante, para un nutrido conjunto de autores (Ellis, 2007; Rescorla, 2002), con muchas probabilidades de evolucionar hacia un trastorno del lenguaje. En consecuencia, se trata de un tópico de investigación de una fuerte actualidad, especialmente porque parece existir una alta probabilidad de que estos hablantes tardíos puedan evolucionar hacia un retraso de lenguaje y convertirse posteriormente en sujetos con TEL (Specific Language ImpairmentSLI). De entre los numerosos autores que se han preocupado por profundizar en este interesante problema se podrían citar, entre muchos otros, a Paul (1991), Rescorla (1989, 1991), Thal y Bates (1988) y Whitehurst y Fischel (1994). Obviamente, el tema tiene una doble trascendencia. En primer lugar, cómo resolver el asunto de la detección temprana y de la prevención; en segundo lugar, cómo solucionar el dilema de llevar a cabo una intervención basada en procedimientos más indirectos (como la estimulación focalizada o el recast conversacional), con estrategias horizontales (trabajando diversos componentes del lenguaje de manera simultánea) y con actividades menos estructuradas, todo esto más orientado a los sujetos con RL o, por el contrario, optar por procedimientos más sistemáticos y regulares (como la imitación), con estrategias verticales (seleccionando objetivos más específicos que afectan a un solo componente del lenguaje) y con actividades fuertemente estructuradas (posición más próxima a los sujetos con TEL).

Esta perspectiva evolutiva de los trastornos del lenguaje ha nacido de la mano de autores del mundo anglosajón (language disorders from a developmental perspective) de la talla de R. Chapman o S. Ellis. Sus 
tesis soportan la idea de la existencia de una secuencia que va de forma inequívoca desde el hablante tardío hacia el TEL, comenzándose a hablar de un espectro de trastorno del lenguaje (Ellis, 2007; Rescorla, 2000, 2002; Rescorla \& Lee, 2000). Esto es, se propone un espectro en el desarrollo de habilidades lingüísticas, como un continuum que evoluciona a través de un proceso basado en la idea de desarmonía (véase también Serra, 2002, p. 65). De este modo, habrá niños que aparentemente gozan de un desarrollo prelingüístico normal, pero que entre los 18 36 meses de edad comienzan a presentar un retraso consistente que evolucionará hacia un TEL (entre los 48-60 meses). Dicho retraso no afectará a un único componente lingüístico, sino que el deterioro en procesos psicolingüísticos básicos y fundamentales para un desarrollo ajustado (como es el caso del almacén fonológico de la memoria de trabajo) provocaría alteraciones en el aprendizaje, primero, del vocabulario y, posteriormente, de los componentes fonológico y morfosintáctico. En consecuencia, no existirá un factor único (por ejemplo, un déficit gramatical) que sea el causante de la existencia de un nivel más bajo de adquisición de destrezas lingüísticas; en su lugar, se hipotetiza que la debilidad en varias habilidades que están al servicio de lenguaje (tales como el procesamiento auditivo, la memoria de trabajo o la planificación motora) pueden desembocar en un empobrecimiento lingüístico. Esta idea es similar a aquella otra que ha venido defendiendo la existencia de múltiples factores subyacentes en el TEL que además pueden diferir de un individuo a otro (Ellis \& Thordardottir, 2002), y por ende no haría sino constatar que estamos ante un trastorno caracterizado por una enorme heterogeneidad.

¿Qué sentido tienen las reflexiones anteriores y hacia dónde nos conducen en este trabajo? Cada vez hay mayor acuerdo en la necesidad de llevar a cabo una identificación temprana de determinados trastornos del lenguaje que permita el diseño e implementación de una intervención diferenciada, prevaleciendo, si se quiere, un interés clínico, a la hora de abordar el estudio de los trastornos en la adquisición del lenguaje. En otras palabras, estaríamos ante el problema de definir el TEL, siguiendo un criterio diagnóstico basado en la severidad del trastorno. Así, aunque resulta evidente que para hablar de un TEL se precisa la existencia de un cierto grado de anomalía con respecto al desarrollo normal, no está ni mucho menos claro cuál debe ser dicho grado de gravedad. Esto ha conducido a ofrecer una visión laxa del problema, en el sentido de incluir dentro del término TEL cualquier tipo de severidad, desde leve a profunda (Ellis, 2007).

En nuestra opinión, la idea del espectro introduce mucha confusión a la hora de hacer un diagnóstico certero y una intervención ajustada y precisa. Desde un punto de vista clínico, habría que distinguir, desde las primeras edades, dos grupos de sujetos que tendrían denominaciones distintas (RL y TEL), ya que la evaluación y la intervención son claramente desiguales. Esto no quiere decir que los sujetos con RL no necesiten de una intervención; al contrario, la requieren, pero su diseño y desarrollo, así como el pronóstico en la evolución del problema, establece principios y expectativas diferentes a lo que se podría pensar, para aplicar a los sujetos con TEL. En muchas ocasiones, los niños con RL solo necesitan un programa de estimulación y/o terapia articulatoria, presentando generalmente un pronóstico óptimo; mientras que los TEL demandan una intervención de mayor intensidad y frecuencia, y tienen un pronóstico más incierto, caracterizado principalmente por la resistencia al cambio.

Con base en esta introducción teórica, se ha diseñado la presente investigación. La realización de un rastreo inicial y de una posterior evaluación en profundidad, permitió detectar niños con problemas de lenguaje, diferenciándose entre RL y TEL. Mientras que los primeros no recibieron intervención alguna, sí se aplicó un programa a los sujetos con TEL. Finalmente, se procedió a realizar un análisis, para valorar la evolución de ambos grupos tanto en el área comprensiva como expresiva.

\section{Objetivos y problemas de investigación}

El objetivo de este estudio fue comprobar la evolución de sujetos con RL (sin recibir tratamiento alguno) y con TEL (sometidos a un tratamiento frecuente e intensivo). El trabajo se desarrolló con sujetos a lo largo de las edades de 3 y 4 años. 
Dicha investigación se llevó a cabo para responder a las siguientes preguntas:

1. ¿Es necesario diferenciar entre RL y TEL?, iSe pueden establecer ambas categorías a partir de una screening?, ¿Cuáles son las características principales que permiten diferenciar entre RL y TEL, en edades tempranas?
2. ¿Puede evolucionar un RL hacia un TEL?

3. ¿Es cierto que los sujetos con TEL presentan una gran resistencia a la intervención?

4. ¿Cuáles serían las implicaciones clínicas de este trabajo para el diagnóstico, la evaluación y la intervención de los trastornos específicos del lenguaje?

\section{TABLA 1}

Cuestionario para la identificación de dificultades en la comunicación, el habla y el lenguaje (Acosta, Moreno, Cámara, Coello $\mathcal{E}$ Mesa, 2007)

\begin{tabular}{|c|c|}
\hline IDENTIFICACIÓN DE DIFICULTADES EN LA COMUNICACIÓN, HABLA Y LENGUAJE & Sí \\
\hline 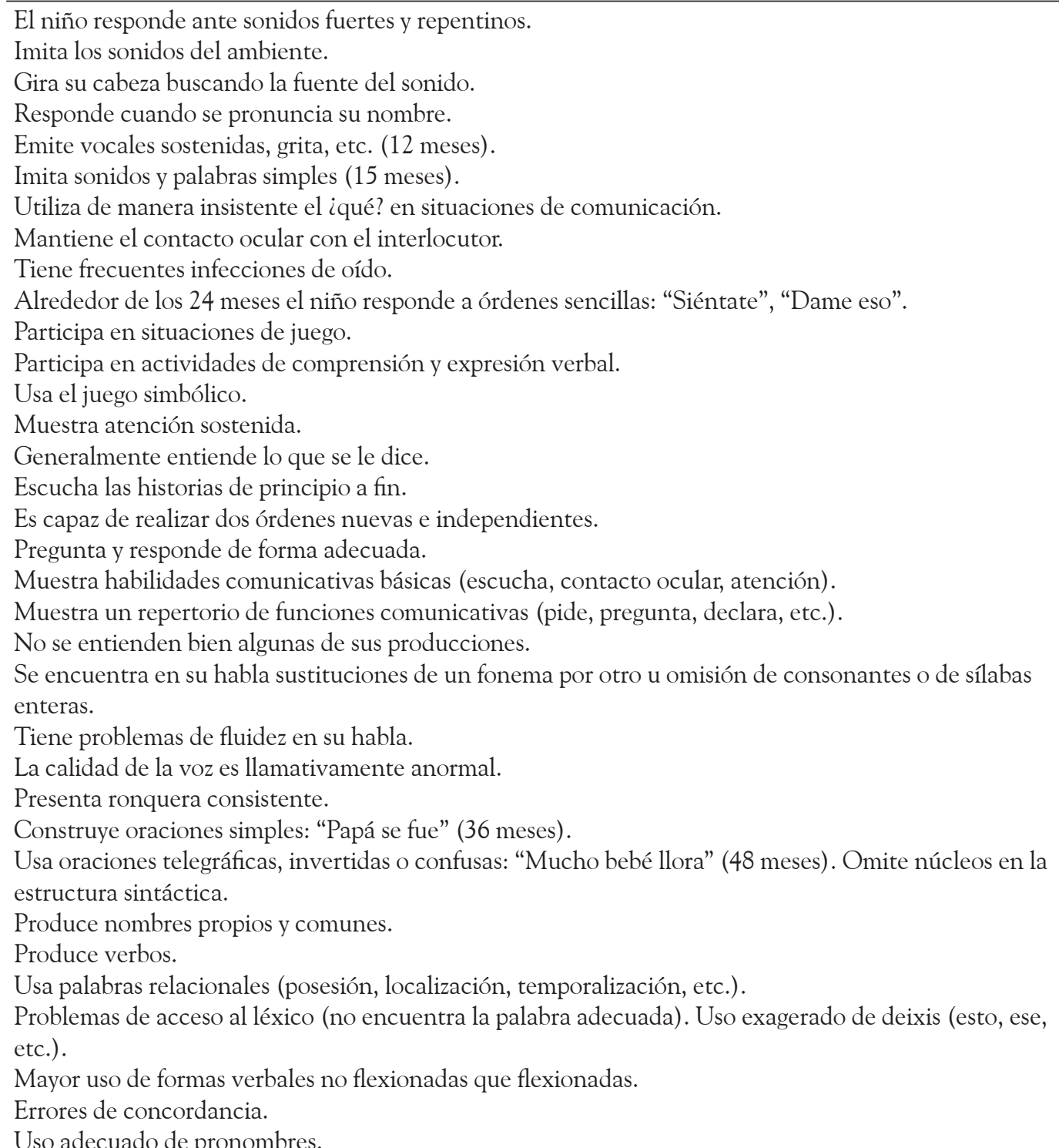 & \\
\hline
\end{tabular}

Fuente: elaboración propia. 


\section{Método}

\section{Selección de la muestra e instrumentos de recolección de la información}

La muestra de sujetos que participaron en el proyecto se obtuvo a partir de un protocolo de observación (véase Tabla 1, Acosta et al., 2007) distribuido a las maestras de Educación Infantil de 3 años en diversos colegios, de varios municipios de la Isla de Tenerife (Islas Canarias, España), en colaboración con los Equipos de Orientación Psicopedagógica de la zona correspondiente. El objetivo de este rastreo era detectar niños y niñas con alguna dificultad de lenguaje. De esta manera se obtuvo, de entre 400 sujetos, un grupo inicial de 12 con algún tipo de retraso entre los 3 y los 4 años. Algunas de las conductas aparecidas fueron: la ininteligibilidad del habla, la no escucha de las narraciones de principio a fin, el uso de oraciones muy telegráficas y las limitaciones de acceso al léxico.

El siguiente criterio de selección consistió en un examen más detallado de los diferentes componentes lingüísticos, utilizándose para ello la Escala de Desarrollo Battelle-BDI (Newborg, Stock \& Weck, 2001) y la Prueba de Lenguaje Oral de Navarra, Revisada, PLON-R (Aguinaga, Armentia, Fraile, Olangua \& Útiz, 2004). El uso de estos dos tests se justifica, el primero de ellos, por la necesidad de tener una valoración general de todas las áreas del desarrollo y, especialmente, certificar la no presencia de alteraciones en el desarrollo cognitivo. La segunda prueba se empleó con la intención de confirmar la existencia de que la dificultad de lenguaje afectaba, al menos, a las dimensiones de contenido (semántica, léxico) y de forma (morfosintaxis y fonología).

A partir de aquí, y con el fin de establecer un criterio de severidad, se diferenció entre RL y TEL. Las características de ambos grupos se muestran en la Tabla 2.

Todos los niños presentaron un desarrollo de los aspectos no verbales dentro de parámetros normales, pero, en cambio, mostraron desajustes en la esfera lingüística, a tenor de los resultados encontrados en la Escala de Desarrollo Battelle-BDI (Newborg et al., 2001). No obstante, esta diferencia inicial necesitaba confirmarse a través de una prueba específica de rendimiento en lenguaje. Para tal fin, se usó la Prueba de Lenguaje Oral de Navarra, Revisada, PLON-R (Aguinaga et al., 2004), que, una vez administrada, arrojó resultados diferenciados. En primer lugar, un total de 6 niños mostraron un retraso significativo en todas las dimensiones del lenguaje (contenido, forma y uso), lo que incluía problemas en vocabulario, morfosintaxis, fonología y pragmática y, en consecuencia, se creyó oportuno que pasaran a una fase de evaluación en profundidad que confirmara o no su diagnóstico como sujetos con TEL. En segundo lugar, los restantes 6 niños manifestaron un cierto retraso en los aspectos formales del lenguaje, esto es, en fonología y morfosintaxis, mientras que las puntuaciones fueron mejores en los aspectos de contenido y uso. Comoquiera que el diagnóstico global que se obtuvo en esta prueba fue el de necesitan mejorar, se consideró oportuno reconocerlos como sujetos con RL.

Posteriormente, se tomó una doble decisión. Primeramente, dejar al grupo de RL sin ningún tipo de actuación, en el sentido de que no se le tomaron más

\section{TABLA 2}

Principales características de los sujetos de la muestra

\begin{tabular}{lcc}
\hline & TRASTORNO ESPECÍFICO DEL LENGUAJE (TEL) & RETRASO LENGUAJE (RL) \\
\hline Edad cronológica & Rango entre 3 y 4 años & Rango entre 3 y 4 años \\
Inteligencia no verbal & Rango 27-41 meses & Rango 26-51 meses \\
Lenguaje & Rango $15-36$ meses & Rango 25-42 meses \\
Sexo & 6 niños & 5 niñas y 1 niño \\
\hline
\end{tabular}

Fuente: elaboración propia. 
datos complementarios ni se les sometió a programa de intervención alguno. El objetivo era verificar si el paso del tiempo -y evidentemente una estimulación natural en los contextos escolar y familiar- provocaba una mejora considerable en su desarrollo lingüístico. Por otra parte, acometer un proceso minucioso de evaluación con el grupo con TEL que verificara el juicio diagnóstico inicial, tal y como se sugiere en la literatura al respecto (Aguado, 1999; Mendoza, 2001). Además de esto, se tomó la decisión de que si se confirmaba el diagnóstico inicial, se les administraría un programa de intervención.

\section{Evaluación de los TEL}

Como se dijo anteriormente, la confirmación de la presencia de sujetos con TEL, especialmente cuando el diagnóstico se quiere obtener en edades tempranas, requiere del suministro de un amplio número de pruebas. Una vez revisados los estudios sobre evaluación del TEL y seleccionados los instrumentos pertinentes, se procedió a un riguroso y largo proceso de evaluación, fruto del cual se obtuvieron los resultados que se expresan en la Tabla 3.

\section{TABLA 3}

Instrumentos utilizados y resultados de la evaluación inicial realizada con el fin de diagnosticar a los sujetos con TEL

\begin{tabular}{ll}
\hline \multicolumn{1}{c}{ Pruebas } & \multicolumn{1}{c}{ Resultados } \\
\hline $\begin{array}{l}\text { Prueba de Lenguaje PLS-4 (Zimmerman, Stei- } \\
\text { ner \& Pond, 2004) }\end{array}$ & $\begin{array}{l}\text { Todos los sujetos estaban entre -1 y -2 desviaciones estándar por } \\
\text { debajo de la media. }\end{array}$ \\
\hline $\begin{array}{l}\text { Escala de Desarrollo Cognitivo General (Mc- } \\
\text { Carthy, 1976) }\end{array}$ & $\begin{array}{l}\text { Desarrollo General Cognitivo, con un rango entre } 73 \text { y } 95 . \\
\text { Todos los sujetos presentaron retraso en el factor verbal, mientras } \\
\text { que el desarrollo no verbal era acorde con su edad. }\end{array}$ \\
\hline $\begin{array}{l}\text { Registro Fonológico Inducido (Juárez \& Mon- } \\
\text { fort, 1996) }\end{array}$ & $\begin{array}{l}\text { Tanto en fonemas como en palabras erróneas, puntuación muy por } \\
\text { debajo de la mínima establecida por el test. }\end{array}$ \\
\hline $\begin{array}{l}\text { Prueba de Discriminación Auditiva (Aguilar \& } \\
\text { Serra, 2003) }\end{array}$ & Por debajo del percentil 50. \\
\hline $\begin{array}{l}\text { Pseudopalabras (Aguado, Cuetos, Domezáin \& } \\
\text { Pascual, 2006) }\end{array}$ & $\begin{array}{l}\text { Resultados muy bajos tanto en palabras familiares como en no } \\
\text { familiares. }\end{array}$ \\
\hline $\begin{array}{l}\text { Imitación de frases (WPPSI, Wechsler, 1970) } \\
\text { Semejanzas (WPPSI, Wechsler, 1970) }\end{array}$ & $\begin{array}{l}\text { Puntuaciones entre el rango } 1 \text { y } 10 \text { muy por debajo de la puntua- } \\
\text { ción inferior del test (11). }\end{array}$ \\
\hline $\begin{array}{l}\text { Puntuaciones entre el rango } 0 \text { y } 6 \text { muy por debajo de la puntua- } \\
\text { ción inferior del test (10). }\end{array}$ \\
\hline $\begin{array}{l}\text { Vocabulario (WPPSI, Wechsler, 1970) } \\
\text { Asociación Visual (ITPA, Kirk, McCarthy \& } \\
\text { Kirk, 1968) }\end{array}$ & $\begin{array}{l}\text { Puntuaciones entre el rango } 0 \text { y } 9 \text { muy por debajo de la puntuación } \\
\text { rior del test, establecido para los } 3 \text { años de edad. }\end{array}$ \\
\hline $\begin{array}{l}\text { Asociación Auditiva (ITPA, Kirk, McCarthy \& } \& \text { rango } 1 \text { y } 10 \text { muy por debajo del límite infe- } \\
\text { Kirk, 1968) }\end{array}$ & $\begin{array}{l}\text { Puntuaciones entre el rango } 3 \text { y } 10 \text { muy por debajo del límite infe- } \\
\text { rior del test, establecido para los } 3 \text { años de edad. }\end{array}$ \\
\hline $\begin{array}{l}\text { Memoria secuencial auditiva (ITPA, Kirk, Mc- } \\
\text { Carthy \& Kirk, 1968) }\end{array}$ & $\begin{array}{l}\text { Puntuaciones entre el rango } 1 \text { y } 4 \text { muy por debajo del límite infe- } \\
\text { rior del test, establecido para los } 3 \text { años de edad. }\end{array}$ \\
\hline $\begin{array}{l}\text { Evaluación de Guiones (Pavez, Coloma \& Ma- } \\
\text { ggiolo, 2008) }\end{array}$ & $\begin{array}{l}\text { Las puntuaciones oscilan entre las de cuatro niños que estarían } \\
\text { dentro del promedio y otros dos que tendrían un desempeño des- } \\
\text { cendido (entre - } 1.5 \text { y - } 2 \text { desviaciones estándar). }\end{array}$ \\
& \\
&
\end{tabular}

Desarrollo narrativo (comprensión y producDéficit narrativo en comprensión y producción. ción del cuento Buenas noches, gorila Peggy, 2001)

Procesamiento fonológico (Jiménez \& Ortiz, Puntuaciones entre los centiles 1-5. 1995) Puntuación Hepta: muy bajo. 


\begin{tabular}{|c|c|}
\hline Pruebas & Resultados \\
\hline $\begin{array}{l}\text { Procesamiento fonológico-PROFON (Lara, } \\
\text { Aguilar \& Serra, 2007) }\end{array}$ & $\begin{array}{l}\text { En los tres niveles de conciencia fonológica (silábico, intrasilábico } \\
\text { y fonémico) no se supera la puntuación cero. } \\
\text { En el conocimiento del nombre de las letras, puntuaciones entre el } \\
\text { rango } 0 \text { y } 5 \text {. } \\
\text { En el conocimiento del sonido de las letras, puntuaciones entre el } \\
\text { rango } 0 \text { y } 5 \text {. }\end{array}$ \\
\hline Análisis morfoléxico & $\begin{array}{l}\text { Índice de diversidad léxico (IDL): rango entre } 0.4 \text { y } 0.7 \text {. } \\
\text { Longitud media de enunciados (LME): rango entre } 0.7 \text { y } 1.5 \text {. }\end{array}$ \\
\hline
\end{tabular}

Fuente: elaboración propia.

Como se puede observar, el perfil obtenido apunta claramente a las características del TEL, debido a una serie de razones que se exponen seguidamente. En primer lugar, la obtención de puntuaciones medias normales en los aspectos no verbales, mientras que el lenguaje se encuentra seriamente comprometido, estando objetivamente representado en una puntuación negativa, en todos los casos, que oscila entre -1 y -2 desviaciones estándar por debajo de la media de sus pares. En segundo lugar, por la presencia de determinados procesos psicolingüísticos afectados como es el caso de la actividad dentro del almacén fonológico de la memoria de trabajo (Aguado, Cuetos, Domezáin \& Pascual, 2006), evaluada a través de repetición de pseudopalabras (Aguado, 2007) y de imitación de frases. Estas características parecen ser una constante en los niños con TEL, sea cualquiera la lengua que hablen. Dicha limitación se manifiesta también en un pobre vocabulario que, en la muestra del presente estudio, coincide con unos resultados muy pobres en el WPSSI, y en la obtención de una bajísima puntuación en el Índice de Diversidad Léxica, conseguida a partir del análisis de una muestra de lenguaje espontánea. De igual forma, y a partir de los 3 años de edad, la afectación del proceso anterior tendrá una incidencia negativa, en primer lugar, en el componente fonológico (véase RFI), con unos resultados desfavorables tanto en la producción de fonemas y palabras como en la discriminación auditiva (véase AREHA), y, en segundo lugar, en el componente morfosintáctico, con la dificultad para adquirir palabras con poca relevancia perceptiva (preposiciones, conjunciones) y con un pobre dominio de las estructuras sintácticas (pobres resultados en PLS-4; puntuaciones muy bajas en la LME). Por último, y aunque son datos que hay que tomar con cierta prudencia por la corta edad de los sujetos, nos encontramos con niños que presentan unas muy pobres habilidades de procesamiento fonológico, así como un déficit en su desarrollo narrativo.

\section{Programa de intervención}

El programa de intervención se desarrolló entre los meses de enero y junio de 2009, iniciándose cuando los niños estaban escolarizados en Educación Infantil de 4 años. Se llevó a cabo un total de 48 sesiones, con una frecuencia de tres veces por semana y una duración de 60 minutos, cada una de ellas. El trabajo se ejecutaba siempre en la primera hora de la mañana, coincidiendo con el espacio horario que las profesoras dedicaban al manejo del lenguaje oral (asamblea, rutinas y narración).

Dicho programa se elaboró después de conocer como era la organización de la enseñanza en cada una de las aulas. A partir de una serie de seminarios y talleres conjuntos, se dio paso al establecimiento de objetivos, contenidos y procedimientos del mismo y se seleccionaron los materiales necesarios para su desarrollo. Una vez que el programa fue bien conocido tanto por las logopedas como por las maestras, se comenzó su implementación colaborativa en las aulas, realizando un seguimiento periódico a través de la observación directa y de entrevistas con las profesoras, con el fin de resolver las dificultades que se iban encontrando y valorar los logros alcanzados.

Los objetivos establecidos en el programa de intervención perseguían el fomento prioritario y regular en el aula de habilidades relacionadas con 
el lenguaje oral y la lectura inicial, tal y como se describe a continuación:

- El diálogo: con el fin de mejorar la participación y la interacción del niño con TEL con sus pares a través de conversaciones sobre lo que hicieron el día anterior o acerca de determinados tópicos que se estuvieran trabajando, por ejemplo, la Unidad Didáctica de La Primavera.

- La representación y verbalización de scripts, con los que se perseguía estimular la secuencia de rutinas familiares; el uso de unidades lingüísticas como el vocabulario, los verbos, los adjetivos, los pronombres y las preposiciones; la formulación de preguntas y la elaboración de frases.

- El desarrollo de habilidades narrativas, a través de la comprensión y la producción de cuentos, a partir de los principios de la lectura dialógica y de un trabajo específico sobre el estímulo de estructuras narrativas basado en el Plan EDEN de Pavez et al. (2008).

- El desarrollo del lenguaje oral, mediante la categorización de conceptos y familias léxicas junto con la distinción de las palabras en distintos tipos de oraciones y de la lectura inicial, esto es, la conciencia metafonológica, el conocimiento del alfabeto y la asociación fonema-grafema.

Los contenidos se organizaron siguiendo un patrón de complejidad creciente. En la Tabla 4 quedan recogidos dichos contenidos diferenciados por áreas de trabajo.

\section{Resultados}

Usando medidas de producción y comprensión de dos tests (Prueba de Lenguaje Oral de Navarra-Revisada, PLON-R de Aguinaga et al., 2004; Prueba de Lenguaje PLS-4 de Zimmerman et al., 2004), el resultado más concluyente es que todos los sujetos diagnosticados con RL en la evaluación inicial evolucionaron favorablemente; en otras palabras, ningún niño etiquetado con RL entre los 3 y los 4 años de edad, tuvo un diagnóstico clínico de TEL cuando se tomaron medidas a los 5 años de edad. Estos datos son consistentes con los de

TABLA 4

Contenidos desarrollados en el programa de intervención

\begin{tabular}{llll}
\hline \multicolumn{1}{c}{ Diálogo } & \multicolumn{1}{c}{ Scripts } & \multicolumn{1}{c}{ Desarrollo narrativo } & \multicolumn{1}{c}{ Lenguaje oral y lectura inicial } \\
\hline -Rutinas diarias como & -La visita al médico. & -Estimulación de guiones & -Definir dibujos. \\
el saludarse, darse los & -El viaje en avión. & (secuencias de acciones & -Clasificar dibujos de distintas \\
buenos días, pasar lista, & -Ir a comer a un & en torno a un personaje, & categorías (alimentos, animales, etc.). \\
hablar del tiempo, etc. & restaurante. & según el Plan EDEN & -Definición oral de palabras. \\
-Iniciar la conversación. & -La visita al veterinario. & (Pavez, et al., 2008). & -Discriminación de sonidos. \\
-Guardar el turno. & -Un día de pesca en un & -Estructura narrativa & -Memoria auditiva. \\
-Formulación de & barquito. & básica (la presentación, & -Lectura dialógica con grandes libros \\
preguntas. & -Un día en la granja. & el episodio y el final, & para llamar la atención sobre el título, \\
-Hablar sobre hechos & -Ir a la peluquería. & según el Plan EDEN & el autor, el copyright, la portada, la \\
pasados. & -Prepararse para ir al & (Pavez et al., 2008). & contraportada, etc. \\
-Hablar acerca de & colegio. & -Lectura de cuentos con & -Lectura dialógica diferenciando las \\
eventos futuros. & un episodio. & imágenes de las palabras. \\
& & & - Explicar y hacer predicciones sobre \\
& & & aspectos que sucederán en la lectura. \\
& & & -Relacionar la lectura con \\
& & & experiencias de los niños. \\
& & & -Reconocimiento de vocales. \\
& & & -Reconocimiento de consonantes.
\end{tabular}


otros estudios que indican que este tipo de retrasos evolutivos, en numerosas ocasiones, muestran un rango de habilidades lingüísticas normales cuando llegan a los 5 años de edad (Paul, 1996; Whitehurst $\&$ Fischel, 1994).

Con el propósito de determinar si hubo diferencias estadísticamente significativas entre los niños con TEL y los que presentaban RL, se efectuó un análisis comparando las puntuaciones obtenidas por los sujetos de ambos grupos, un año después de haber sido evaluados y diagnosticados con las etiquetas anteriores. En esta ocasión se eligió la versión española del PLS-4, considerada una prueba potente para el análisis de los aspectos comprensivos y expresivos del lenguaje. Además, el uso de este test permitió la obtención de puntuaciones totales objetivas. Dadas las características de la muestra estudiada, se recurrió a estadísticos no paramétricos, y más concretamente se utilizó la U de Mann-Whitney. En la Tabla 5 se recogen los datos más importantes.

Las puntuaciones de la muestra en esta segunda evaluación difieren mucho por el hecho de su pertenencia al grupo con TEL o con RL. A la luz de estos primeros resultados, se observa la existencia de una clara disparidad en el rendimiento, tanto en expresión como en producción; además, estas diferencias resultan significativas si atendemos al valor de Z. Téngase en cuenta, igualmente, que los sujetos del primer grupo recibieron un programa de intervención, mientras que los del segundo, estuvieron a expensas del efecto evolutivo, del paso del tiempo y de las situaciones de interacción y aprendizaje habituales tanto en el aula como en el hogar.

Comoquiera que se recomienda la aplicación de otras pruebas de lenguaje que pudieran contrastar los resultados anteriores, se procedió de la misma manera pero utilizando ahora la Prueba de Lenguaje Oral de Navarra Revisada-PLON-R (Aguinaga et al., 2004). En esta ocasión, y empleando de nuevo la $U$ de Mann-Whitney, se obtuvieron resultados todavía más claros que en la ocasión anterior, tal y como se recoge en la siguiente (Tabla 6).

Como puede verse, se encuentran diferencias estadísticamente significativas entre ambos grupos. En esta dirección, hay que destacar una evolución siempre favorable de los sujetos con RL, sin que sean sometidos a intervención alguna. Y lo que resulta más importante, los resultados obtenidos apuntan a que no tiene porqué haber un recorrido desde el RL

\section{TABLA 5}

Contraste utilizando la $U$ de Mann-Whitney entre las puntuaciones alcanzadas por los sujetos con TEL y con RL, en la Prueba de Lenguaje PLS-4

\begin{tabular}{lccccc}
\hline & $\begin{array}{c}\text { Rangos } \\
\text { Medios TEL }\end{array}$ & $\begin{array}{c}\text { Rangos } \\
\text { Medios RL }\end{array}$ & $\begin{array}{c}\text { U de Mann- } \\
\text { Whitney }\end{array}$ & $z$ & Sig. \\
\hline $\begin{array}{l}\text { PLS-4. Desviación } \\
\begin{array}{l}\text { Estándar } \\
\text { Puntuación } \\
\text { Total }\end{array}\end{array}$ & 4.5 & 8.5 & 6 & $-1.963^{*}$ & $0.05^{*}$ \\
\hline
\end{tabular}

$* p \leq 0.05$

Fuente: elaboración propia.

TABLA 6

Contraste utilizando la U de Mann-Whitney entre las puntuaciones alcanzadas por los sujetos con TEL y con RL, en el PLON

\begin{tabular}{lccccc}
\hline & $\begin{array}{c}\text { Rangos } \\
\text { Medios TEL }\end{array}$ & $\begin{array}{c}\text { Rangos } \\
\text { Medios RL }\end{array}$ & $\begin{array}{c}\text { U de Mann- } \\
\text { Whitney }\end{array}$ & $z$ & Sig. \\
\hline PLON-R. Puntuación total & 4 & 9 & 3 & -2.739 & $0.006^{* *}$ \\
\hline
\end{tabular}

$* * \mathrm{p} \leq 0.01$

Fuente: elaboración propia. 
hasta el TEL, tratándose de categorías diagnósticas diferenciadas, tanto en el plano de la evaluación como en el de la intervención. Por lo tanto, la idea de espectro debería cuestionarse y revisarse, tal y como se abordará en la discusión de este trabajo.

Con todo, se necesita de una mayor actividad investigadora en este tópico y, evidentemente, estudios longitudinales que incorporen una muestra más representativa.

\section{Discusión}

El principal interés de este trabajo se encuentra en la dimensión clínica. En efecto, conseguir un diagnóstico diferencial, permitirá afinar más en el diseño del plan de intervención, especialmente a la hora de organizar bien sus objetivos, procedimientos, estrategias y actividades, tanto como la frecuencia e intensidad del trabajo y los contextos, y los agentes de la intervención.

En consecuencia, las expresiones hablante tardío (late talkers) y retraso del lenguaje (language delay) no tienen unas connotaciones clínicas, si atendemos a un criterio de severidad o gravedad y de prevalencia en el tiempo, sin embargo, ocurre todo lo contrario cuando nos referimos a un TEL. Desde un punto de vista de lo que constituye la detección temprana, hay que tener en cuenta que los hablantes tardíos y los RL pueden identificarse con mayor precocidad, entre los $18-20$ y $24-36$ meses, respectivamente, mientras que sigue siendo muy difícil diagnosticar con garantías un TEL antes de la edad de 4 años (Rescorla \& Lee, 2000). Al mismo tiempo habrá que señalar un mayor nivel de profundización en la evaluación confirmatoria del TEL, ya que es necesario usar instrumentos cualitativos y cuantitativos, y la búsqueda de resultados en cada uno de los componentes del lenguaje, así como de procesos psicolingüísticos y neuropsicológicos que pudieran estar afectados, tal y como ya se ha comentado en la introducción de este trabajo.

Se sabe que entre un 5 y un $10 \%$ de niños y niñas empezaron el lenguaje después de los 24 meses, y alguno de ellos lo iniciarán todavía más tarde y de forma muy pobre, constituyendo claramente un grupo de riesgo para su futuro desarrollo cognitivo, escolar y social. De entre todos ellos, solo un $0.3 \%$ evolucionan hacia un TEL (Serra, 2002), mientras que el resto hace otro tipo de recorridos, entre ellos, derivan en un RL o hacia un problema de habla. Hablar en términos de espectro de trastorno del lenguaje, incluyendo ambos problemas dentro de un continuun puede resultar equívoco, y de una gran confusión de cara a establecer programas de trabajo preventivos y tempranos.

Un RL se caracteriza por presentar dificultades en la adquisición y el desarrollo del lenguaje, sin que curse con otros problemas de carácter sensorial, orgánico, cognitivo o conductual (Belinchón, Rivière \& Igoa, 1992) que puede afectar a varios componentes del lenguaje y se caracteriza por una aparición tardía en relación con lo que se considera el patrón habitual de adquisición lingüística. En consecuencia, los niños con RL muestran patrones lingüísticos correspondientes a niños de menor edad (Moreno, Suárez, Martínez \& García-Bahamonde, 2004). Buena parte de estos RL se suele explicar por causas derivadas de un retraso madurativo $\mathrm{y} / \mathrm{o}$ factores socioculturales o de entorno lingüístico (estilos comunicativos de la familia, prácticas de alfabetización temprana en el hogar, etc.). Si se analiza con detenimiento lo anterior, se puede pensar en algunas causas externas, ajenas a los propios sujetos como desencadenantes del retraso; dentro de ellas convendría señalar los estilos comunicativos de los padres, los patrones de interacción entre los diferentes miembros de la familia (por ejemplo, la existencia de hermanos y el lugar que se ocupa entre ellos) y el impacto que supone la transición de la familia a la escuela, y en relación con esta circunstancia, el tipo y la cantidad de apoyo que se reciba en el contexto del aula. Pudiera pensarse, entonces, que la intervención estuviera organizada en torno a una estimulación focalizada en el niño y sobre los interlocutores que interactúan con él, en los contextos escolar y familiar.

Por el contrario, los sujetos con TEL también tienen un problema de adquisición y desarrollo del lenguaje, que no se explica por causas cognitivas, neurológicas ni, lo que resulta más importante, por factores socioambientales. Hay además tres criterios específicos que hay que tener en cuenta: 
una puntuación en los tests de lenguaje con más de una desviación típica por debajo de la media; un cociente intelectual no inferior a 85 y la perdurabilidad en el tiempo y resistencia a la recuperación con el tratamiento. Consiguientemente, estamos ante sujetos que, usualmente, presentan patrones distorsionados de lenguaje -como ya se dijo en la introducción de este trabajo-, y que mantienen una diferencia respecto a los RL en la presentación de una evolución mucho más lenta y su resistencia al cambio, a pesar de que, como ha ocurrido en el presente estudio, se sometan a un programa de intervención frecuente e intenso.

Descendiendo a un nivel más concreto de análisis, hay algunas investigaciones sobre la diferenciación entre RL y TEL. En primer lugar, estarían aquellos trabajos que inciden en que los TEL tienen problemas con los elementos menos salientes del lenguaje a causa de un déficit general de procesamiento (Leonard, 1998). En segundo lugar, se hace referencia a su déficit en la memoria de trabajo fonológica que ocasiona una limitación en mantener o almacenar los elementos del lenguaje en la memoria temporal (Montgomery, 1995). En tercer lugar, se menciona el déficit en procesamiento temporal, por el cual hay dificultades en la percepción de estímulos rápidos o de varios estímulos al mismo tiempo (Tallal, 1999). Estas tres hipótesis mantienen una conexión clara con la fonología de los TEL. Así, la omisión de marcas de flexión está relacionada con su coincidencia con sílabas átonas iniciales -en inglés-, se produce una omisión de sílabas átonas en palabras largas y una omisión de las sílabas átonas pretónicas debido a que son mucho más rápidas. En un trabajo de Aguilar (2002) se revisan muchas investigaciones en las que se da cuenta de que los sujetos con TEL muestran perfiles diferentes en el nivel de inteligibilidad, en el inventario fonético, en el porcentaje de consonantes correctas y en el tipo de procesos. Esta misma autora propone hacer un diagnóstico diferencial precoz, a partir del análisis de los procesos de simplificación fonológica. Más concretamente, señala que la característica más determinante a la hora de diferenciar el TEL y el $\mathrm{RL}$, consiste en que los primeros presentan muchas omisiones de sílabas átonas (elementos más cortos y menos salientes del lenguaje), especialmente en posición inicial, debido a un problema de procesamiento. Para esta autora, hay unas implicaciones claras ya que:

La mayoría de las palabras función en español (determinantes, preposiciones, pronombres clíticos, etc.) son monosílabos átonos antepuestos a las palabras contenido (verbos, nombres, adjetivos, etc.), convirtiéndose de esta manera en sílabas átonas iniciales de estas palabras. Por lo tanto, al igual que las sílabas átonas pertenecientes a las palabras, también estas sílabas serán omitidas, afectando de este modo la fonología a la morfosintaxis (Aguilar, 2002, p. 96).

Otro dato coincidente con nuestra investigación es el tema del sexo. En algunos estudios epidemiológicos (Law, Boyle, Harris, Harkness \& Nye, 2000; Mendoza, 2001) se confirma la mayor prevalencia en niños que en niñas, con una proporción de 2:1. En el presente estudio, los seis casos de TEL se corresponden con niños; por el contrario, la prevalencia en el RL, es mayoritariamente de niñas, con una proporción de 5:1.

Estamos, por lo tanto, en la idea de que RL y TEL pueden identificarse tempranamente, y que se trata de categorías clínicas que se van a distinguir principalmente por su nivel de severidad o gravedad. En definitiva, se debe hablar de dos categorías distintas, cuya diferenciación tiene un carácter técnico y orientativo para la intervención (Aguado, 1993, 1995; Monfort \& Juárez, 1993).

Se puede inferir a partir de este estudio -con todas las precauciones que hay que tener debido a las limitaciones de la muestra con la que se ha trabajado- que la intervención temprana será un éxito en los niños con RL, pero que en el caso de los TEL, la resistencia al cambio será mayor, debiendo prolongarse la ayuda durante mucho más tiempo, lo que hará coincidir este trastorno con la aparición, más que probable, de ciertas dificultades de aprendizaje, especialmente aquellas que afectan a la lectura (Stackhouse \& Wells, 1997, 2000). 


\section{Conclusiones}

A partir de este estudio se pueden establecer algunas conclusiones, al objeto de seguir incentivando la investigación necesaria para llegar a afirmaciones mucho más contrastadas. En cualquier caso, en primer lugar, parece haber una clara diferencia diagnóstica entre los sujetos con RL y TEL. La mayor severidad del trastorno junto con una afectación de procesos psicolingüísticos fundamentales, diferencia el TEL de los RL.

En segundo lugar, queda claro que los sujetos con RL, no solo no evolucionan hacia un TEL, sino que su propio desarrollo evolutivo, junto con el aprendizaje realizado en los contextos de la casa y la escuela, favorece un progreso similar al de sus pares, a la edad de 5 años.

En tercer lugar, la intervención con los TEL no ha provocado los beneficios esperados inicialmente. Se trata de un trastorno que ofrece una evidente resistencia al cambio.

Por último, y considerando que la intervención ha estado basada en el aula, con el uso de procedimientos muy orientados a la facilitación de la comprensión de las tareas y del lenguaje utilizado -uso de modelado interactivo y andamiaje-, podría resultar interesante reflexionar sobre la necesidad de plantear otro tipo de acciones y contextos que pudieran fomentar la adquisición de habilidades específicas en estos sujetos.

\section{Referencias}

Acosta, V., Moreno, A., Cámara, M., Coello, A. \& Mesa, J. (2007). Guía de actuaciones educativas en el ámbito de la comunicación y el lenguaje. Canarias: Consejería de Educación, Cultura y Deportes, Gobierno de Canarias.

Aguado, G. (1995). Estrategias para la intervención en los retrasos del lenguaje. En M. Monfort (Ed.), Enseñar a hablar (pp. 235-258). Madrid: CEPE.

Aguado, G. (1993). Retardo del lenguaje. En J. Peña (Ed.), Manual de logopedia (pp. 199-215). Barcelona: Masson.
Aguado, G. (1999). Trastorno específico del lenguaje. Retraso de lenguaje y disfasia. Archidona, Málaga: Aljibe.

Aguado, G. (2007). Apuntes acerca de la investigación sobre el TEL. Revista de Logopedia, Foniatría y Audiología, 27(3), 103-109.

Aguado, G., Cuetos, F., Domezáin, M. \& Pascual, B. (2006). Repetición de pseudopalabras en niños españoles con trastorno específico del lenguaje: marcador psicolingüístico. Revista de Neurología, 43 (Suppl. 1), 201-208.

Aguilar, E. (2002). Diagnóstico diferencial precoz entre el Trastorno del Lenguaje y el Retraso del Lenguaje a partir de los procesos de simplificación fonológica. Revista de Logopedia, Foniatría y Audiología, 22(2), 90-99.

Aguilar, E. \& Serra, M. (2003). A-RE-HA. Análisis del retraso del habla. Barcelona: Edicions Universitat de Barcelona.

Aguinaga, G., Armentia, M., Fraile, A., Olangua, P. \& Útiz, N. (2004). Prueba de Lenguaje Oral de Navarra-Revisada (PLON-R). Pamplona: Fondo de Publicaciones del Gobierno de Navarra.

Belinchón, M., Rivière, A. \& Igoa, J. (1992). Psicología del lenguaje. Investigación y Teoría. Madrid: Trotta.

Bishop, D. (1997). Uncommon understanding. Hove, UK: Psychology Press.

Ellis, S. (2007). Language development spectrum? En R. Paul (Ed.), Language disorders from a developmental perspective (pp. 83-101). Mahwah, NJ: Erlbaum.

Ellis, S. \& Thordardottir, E. (2002). Cognition and language. En P. Acardo, A. Capute \& B. Rogers (Eds.), Disorders of language development (pp. 2137). Timoniun, MD: York.

Hincapié, L., Giraldo, M., Lopera, F., Pineda, D., Castro, R., Lopera, J. et al. (2008). Trastorno Específico del Desarrollo del Lenguaje en una población infantil colombiana. Universitas Psychologica, 7(2), 557-569.

Jiménez, J. \& Ortiz, R. (1995). Conciencia fonológica y aprendizaje de la lectura: teoría, evaluación e intervención. Madrid: Síntesis.

Juárez, A. \& Monfort, M. (1996). Registro Fonológico Inducido. Madrid: CEPE.

Kirk, S., McCarthy, D. \& Kirk, W. (1968). Test Illinois de Habilidades Psicolingüísticas. Madrid: TEA. 
Lara, M., Aguilar, E. \& Serra, M. (2007). Prueba de Procesamiento Fonológico (PROFON). Bogotá: Universidad Nacional de Colombia.

Law, J., Boyle, J., Harris, F., Harkness, A. \& Nye, C. (2000). Prevalence and natural history of primary speech and language delay: Findings from a systematic review of the literature. International Journal of Language and Communication Disorders, 35, 165-188.

Leonard, L. (1998). Children with specific language impairment. Massachusetts: MIT Press.

McCarthy, D. (1976). Escalas McCarthy de Aptitudes y Psicomotricidad para niños. Madrid: TEA.

Mendoza, E. (Coord.). (2001). Trastorno Específico del Lenguaje (TEL). Madrid: Pirámide.

Monfort, M. \& Juárez, A. (1993). Los niños disfásicos. Descripción y tratamiento. Madrid: CEPE.

Montgomery, J. (1995). Sentence comprehension in children with Specific Language Impairment: The role of phonological working memory. Journal Speech and Hearing Research, 38, 187-199.

Moreno, J., Suárez, A., Martínez, J. \& García-Bahamonde, M. (2004). Retrasos en la adquisición y desarrollo del lenguaje. Estudio de casos. Madrid: Eos.

Newborg, J., Stock, J. \& Weck, L. (2001). Inventario de Desarrollo Battelle. Madrid: TEA.

Pavez, M., Coloma, C. \& Maggiolo, M. (2008). El desarrollo narrativo en niños. Barcelona: Ars Médica.

Paul, R. (1991). Profiles of toddlers with slow expressive language development. Topics in Language Disorders, 11, 1-13.

Paul, R. (1996). Clinical implications of the natural history of slow expressive language development. American Journal of Speech-Language Pathology, 5, 5-21.

Peggy, R. (2001). Buenas noches, gorila. Nueva York: Putnan's.

Pérez, E. \& Serra, M. (1998). Análisis del retraso del lenguaje. Barcelona: Ariel Practicum.

Rescorla, L. (1989). The Language Development Survey: A screening tool for delayed language in toddlers. Journal of Speech and Hearing Disorders, 54, 587-599.
Rescorla, L. (1991). Identifying expressive delay at age two. Topics in Language Disorders, 11, 14-20.

Rescorla, L. (2000). Do late-talking toddlers turn out to have reading difficulties a decade later? Annals of Dyslexia, 50, 87-102.

Rescorla, L. (2002). Language and reading outcomes to age 9 in late-talking toddlers. Journal of Speech, Language, and Hearing Research, 45, 360-371.

Rescorla, L. \& Lee, E. (2000). Language Impairment in young children. En T. Layton \& L. Watson (Eds.), Handbook of early language impairment in children. Nature (Vol. 1, pp. 1-38). Nueva York: Delmar.

Serra, M. (1997). Dificultades cognitivas y lingüísticas en los niños con TEL. Revista de Logopedia, Foniatría y Audiología, 27(2), 79-91.

Serra, M. (2002). Trastornos del lenguaje: preguntas pendientes en investigación e intervención. Revista de Logopedia, Foniatría y Audiología, 22(2), 63-76.

Serra, M. \& Conti-Ramsden, G. (1998). Retraso y Trastorno Específico del Lenguaje (Disfasia): diagnóstico diferencial e intervención. Actas del XX Congreso Internacional de AELFA, Barcelona, 40-45.

Stackhouse, J. \& Wells, B. (1997). Children's speech and literacy difficulties 1. A psycholinguistic framework. Londres: Whurr Publishers.

Stackhouse, J. \& Wells, B. (2000). Children's speech and literacy difficulties. Identification and intervention. Londres: Whurr Publishers.

Tallal, P. (1999). Children with Language Impairment can be accurately identified using temporal processing measures: A response to Zhang and Tomblin. Brain and Language, 65, 395-403.

Thal, D. \& Bates, E. (1988). Language and gesture in late talkers. Journal of Speech and Hearing Research, 34, 604-612.

Wechsler, D. (1970). Test de inteligencia para preescolares. Madrid: TEA.

Whitehurst, G. \& Fischel, J. (1994). Early developmental language delay: What, if anything, should the clinician do about it? Journal of Child Psychology and Psychiatry, 35, 613-648.

Zimmerman, I., Steiner, V. \& Pond, R. (2004). Preschool Language Scale-Spanish-4 (PLS-4). Chicago: Psychological Corporation. 
